\title{
Coupling of lateral grating displacement to the output ports of a diffractive Fabry-Perot cavity
}

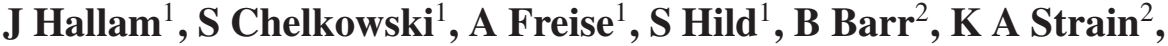 \\ O Burmeister ${ }^{3}$ and R Schnabel ${ }^{3}$ \\ ${ }^{1}$ School of Physics and Astronomy, University of Birmingham, Edgbaston, \\ Birmingham B15 2TT, UK \\ ${ }^{2}$ Institute for Gravitational Research, Department of Physics and Astronomy, University of \\ Glasgow, Glasgow G12 8QQ, UK \\ ${ }^{3}$ Max-Planck-Institut für Gravitationsphysik (Albert-Einstein-Institut) and Leibniz Universität \\ Hannover, Callinstraße 38, D-30167 Hannover, Germany \\ E-mail: jmh@star.sr.bham.ac.uk
}

Received 19 March 2009, accepted for publication 13 May 2009

Published 2 June 2009

Online at stacks.iop.org/JOptA/11/085502

\begin{abstract}
Diffraction gratings have been proposed as core elements in future laser-interferometric gravitational wave detectors. In this paper, we use a steady-state technique to derive coupling of lateral grating displacement to the output ports of a diffractive Fabry-Perot cavity. By introducing a signal to noise ratio (SNR) for each of the three cavity output ports, the magnitudes of the noise sidebands originating from lateral grating displacement are compared to the magnitude of a potential gravitational wave signal. For the example of a $3 \mathrm{~km}$ long Fabry-Perot cavity featuring parameters similar to the planned Advanced Virgo instrument, we found that the forward-reflecting grating port offers the highest SNR at low frequencies. Furthermore, for this example suspension requirements for lateral isolation were computed, and a factor of 20 relaxation at a frequency of $10 \mathrm{~Hz}$ can be gained over the transmitted port by observing the forward-reflected port.
\end{abstract}

Keywords: gravitational wave detector, diffraction grating, interferometry

(Some figures in this article are in colour only in the electronic version)

\section{Introduction}

It has been suggested that the sensitivity of high-precision interferometers, with particular reference to laser-interferometric gravitational wave detectors, could be improved by using an all-reflective optical configuration [1]. Expected improvements include eliminating transmissive thermal noise and freeing optical substrates to be opaque, thus widening the range of material choices. Furthermore, the shot-noise contribution can be reduced by increasing laser power without increasing technical problems such as thermal lensing. Diffractive optics have been suggested as possible replacements for mirrors and beam-splitters that could function in an all-reflective configuration [2]. This document considers the case where a diffractive optic is used in place of a mirror to couple light into a FabryPerot cavity [3].

It has been found that even state of the art diffraction gratings are too low in diffraction efficiency to allow highfinesse cavities based on the grating diffraction [4, 5]. An alternative is to use a diffraction grating with low diffraction efficiency as an incoupler to a Fabry-Perot cavity, because in this case there is the advantage that the cavity finesse is determined by the reflection efficiency of the grating. In three-port, second-order Littrow configuration (as shown in figure 3) it is the zeroth-order diffraction (equivalent to the reflection) that retains the light field in the Fabry-Perot cavity [3]. Standard dielectric coating techniques provide the high-reflectance efficiency required for such a configuration. 


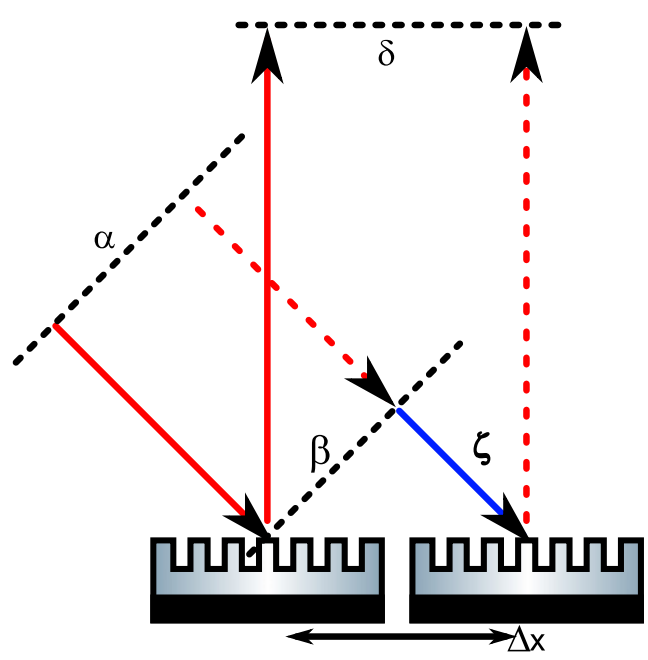

Figure 1. Wavefronts coupling through a diffraction grating. The wavefront (propagation vector shown in solid red) originates at $\alpha$, travels to $\beta$ and is diffracted to $\delta$. When the grating is laterally displaced by $\Delta x$, the wavefront (propagation vector shown red dashed) must pass through additional path-length $\zeta$ (propagation vector shown in solid blue).

\section{Phase noise originating from lateral oscillation of the grating}

In comparison to a standard Fabry-Perot cavity featuring a transmissive input mirror, the diffractive nature of a grating causes an additional coupling of geometry changes into phase noise [6]. This phase noise stems purely from the geometry of the optics, and can be fully calculated from the grating equation and the geometry of the set-up alone. Freise et al determined that, assuming a sensitivity goal of $h=10^{-23} \mathrm{~Hz}^{-1 / 2}$, Virgo-like interferometer parameters [7] and typical values for the grating parameters, a standard cavity required end-mirror angular alignment of $\gamma<2 \times 10^{-16} \mathrm{rad} \mathrm{Hz}^{-1 / 2}$ whereas a three-port-coupled grating cavity required $\gamma<1 \times 10^{-21} \mathrm{rad} \mathrm{Hz}^{-1 / 2}$, needing a five orders of magnitude improvement in the end turning mirror alignment for equivalent insensitivity to this noise source. Improvement in alignment of the injection optics and lateral grating suspension would also be required.

In this paper we consider the similar case of grating displacement perpendicular to the beam and across the grating corrugation (lateral displacement) which couples into phase noise. The phase change resulting from the displacement is due to a path-length difference $\zeta$ between parallel wavefronts, shown for a simple geometrical case in figure 1 .

\section{Frequency domain modelling of phase noise coupling in a grating cavity}

In order to quantitatively analyse the coupling of the phase noise introduced by grating displacement to the output ports of a diffractive cavity we perform a frequency domain analysis. By carrying out this analysis for the effect of a potential gravitational wave signal and the noise effect of lateral grating displacement we can obtain the signal to noise ratio (SNR) at all three output ports of the grating cavity. The aim is to find the port with the best SNR.

To compare the signal to noise ratio at the different output ports we first determine the grating coupling relations. We then review the mechanism of frequency sideband generation by a laterally displaced grating and identify the complex field amplitudes at the sideband frequency caused by (and in terms of) each carrier light field incident on the grating. Using the coupling relations we calculate each carrier light field incident on the grating in terms of a single external input carrier field. These are then used to determine the amplitude of the input sideband fields. Again utilizing the coupling relations we obtain the amplitude of the sideband fields at the output ports. We next derive the amplitude of the potential gravitational wave signal sidebands at the output ports and divide by the grating displacement noise sidebands to obtain the signal to noise ratio.

The typical expression for an electromagnetic light field is $E=a_{q} \mathrm{e}^{-\mathrm{i} \omega t}$, where $a_{q}$ is the field amplitude (which may be a complex number) with $q$ a numeric subscript denoting location in the optical system consistent with figure 3 and $\omega$ is the angular frequency of the light field. For coupling relations we will use $a_{q}$ instead of $E$. The frequency $\omega$ of a field described by an amplitude $a_{q}$ is determined by the context.

\subsection{Coupling relations of a static grating}

The coupling relations of a single, static grating are given in [8]:

$$
\left(\begin{array}{l}
a_{4} \\
a_{1} \\
a_{3}
\end{array}\right)=\left[\begin{array}{ccc}
\eta_{2} \mathrm{e}^{-\mathrm{i} \phi_{2}} & \eta_{1} \mathrm{e}^{-\mathrm{i} \phi_{1}} & \eta_{0} \\
\eta_{1} \mathrm{e}^{-\mathrm{i} \phi_{1}} & \rho_{0} & \eta_{1} \mathrm{e}^{-\mathrm{i} \phi_{1}} \\
\eta_{0} & \eta_{1} \mathrm{e}^{-\mathrm{i} \phi_{1}} & \eta_{2} \mathrm{e}^{-\mathrm{i} \phi_{2}}
\end{array}\right]\left(\begin{array}{c}
b_{0} \\
a_{2}^{\prime} \\
0
\end{array}\right),
$$

where $\eta$ is the amplitude coefficient and $\phi$ is the constant phase change associated with diffraction in the order given by the subscript. Bunkowski et al [8] have shown that positive and negative diffraction orders have the same $\eta$ and $\phi$ in this configuration so only positive subscripts will be used. Zeroth order diffraction is reflection and $\rho_{0}$ is the amplitude coefficient in the special case of zeroth order diffraction normal to the surface of the grating. Adopting simplified notation we can write

$$
\left(\begin{array}{l}
a_{4} \\
a_{1} \\
a_{3}
\end{array}\right)=\left[\begin{array}{ccc}
G_{2} & G_{1} & G_{0} \\
G_{1} & \rho_{0} & G_{1} \\
G_{0} & G_{1} & G_{2}
\end{array}\right]\left(\begin{array}{c}
b_{0} \\
a_{2}^{\prime} \\
0
\end{array}\right) .
$$

\subsection{Coupling relations of an oscillating grating}

Consider the case of an oscillating grating with two incident light fields shown in figure 2: in case A the external input field with amplitude $b_{0}$ and in case B the field with amplitude $a_{2}^{\prime}$. The frequency sideband generation for a grating oscillating with angular frequency $\omega_{m}$ and modulation index $m$ will be demonstrated for output $a_{1}$ in case A. The oscillating grating causes phase modulation of the diffracted fields [6]:

$$
a_{1}=b_{0} G_{1} \mathrm{e}^{-\mathrm{i} m \cos \left(\omega_{m} t\right)} .
$$

The complex field amplitude resulting can be expanded using the Bessel function $J_{k}(m)[9,10]$. In the case $m \ll 1$ it is 

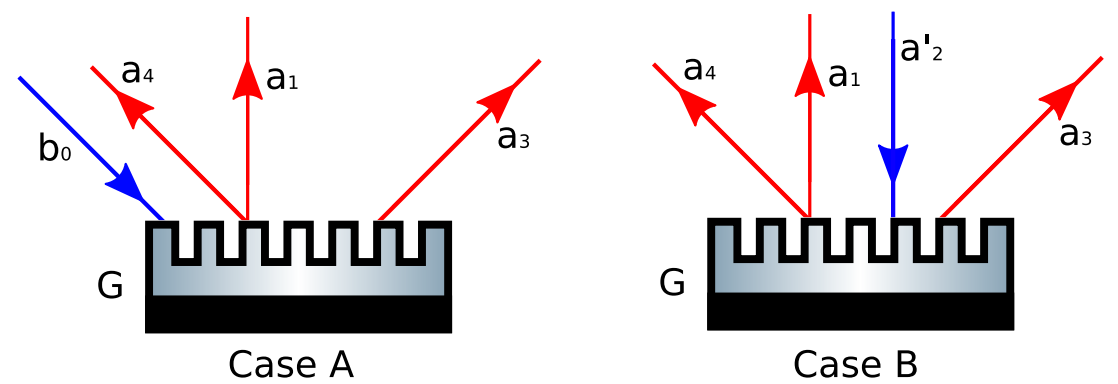

Figure 2. Input ( $b_{0}, a_{2}^{\prime}$, shown blue) and output $\left(a_{1}, a_{3}, a_{4}\right.$, shown red) light field amplitudes at a phase modulating grating in second order Littrow configuration in case A, and zeroth order Littrow configuration in case B (labels consistent with figure 3).

sufficient to consider terms linear in $m$. It follows that only $k=-1,0,1$ need to be considered, resulting in coefficients

$$
J_{1}(m)=m / 2, \quad J_{0}(m)=1, \quad J_{-1}(m)=-m / 2,
$$

thus obtaining

$$
\begin{gathered}
a_{1}=b_{0} G_{1}\left(J_{0}(m)+\mathrm{i} J_{1}(m) \mathrm{e}^{\mathrm{i} \omega_{m} t}-\mathrm{i} J_{-1}(m) \mathrm{e}^{-\mathrm{i} \omega_{m} t}\right) . \\
a_{1}=b_{0} G_{1}\left(1+\mathrm{i} \frac{m}{2} \mathrm{e}^{\mathrm{i} \omega_{m} t}-\mathrm{i} \frac{m}{2} \mathrm{e}^{-\mathrm{i} \omega_{m} t}\right) .
\end{gathered}
$$

When a grating is laterally oscillated to a maximum displacement of $\Delta x$ the modulation index is given by $m=2 \pi \Delta x \mu / d$, where $d$ is the corrugation period of the grating and $\mu$ is the diffraction order, resulting in [6]

$$
a_{1}=b_{0} G_{1}\left(1+\mathrm{i} \frac{\pi \Delta x \mu}{d} \mathrm{e}^{\mathrm{i} \omega_{m} t}-\mathrm{i} \frac{\pi \Delta x \mu}{d} \mathrm{e}^{-\mathrm{i} \omega_{m} t}\right) .
$$

The terms including $\mathrm{e}^{ \pm \mathrm{i} \omega_{m} t}$ are called sidebands. In the following we will compute the sideband amplitudes in a cavity with the grating as the input coupler. The amplitudes in front of the $\mathrm{e}^{ \pm \mathrm{i} \omega_{m} t}$ as computed in equation (7), when coupled through the grating, are the sideband amplitudes for output $a_{1}$ in case A $[11,12]$. First order diffraction $(\mu=1)$ from the grating is the coupling between $b_{0}$ and $a_{1}$ :

$$
\mathrm{i} b_{0} \frac{\pi \Delta x}{d} G_{1} \text {. }
$$

Having demonstrated the calculation of the sideband amplitude for output $a_{1}$ in case A we will now write the result for outputs $a_{3}$ and $a_{4}$ in case A using the same method. Zeroth order diffraction ( $\mu=0$ ) occurs between $b_{0}$ and $a_{3}$ and therefore no sidebands are generated. Second order diffraction $(\mu=2)$ occurs between $b_{0}$ and $a_{4}$, so the sideband amplitude obtained is

$$
\mathrm{i} b_{0} \frac{2 \pi \Delta x}{d} G_{2} \text {. }
$$

Having dealt with case A we proceed to case B in figure 2. Zeroth order diffraction ( $\mu=0$ ) occurs between $a_{2}^{\prime}$ and $a_{1}$ and therefore no sidebands are generated. Negative first order diffraction $(\mu=-1)$ occurs between $a_{2}^{\prime}$ and $a_{3}$, so the sideband amplitude obtained is

$$
-\mathrm{i} a_{2}^{\prime} \frac{\pi \Delta x}{d} G_{1}
$$

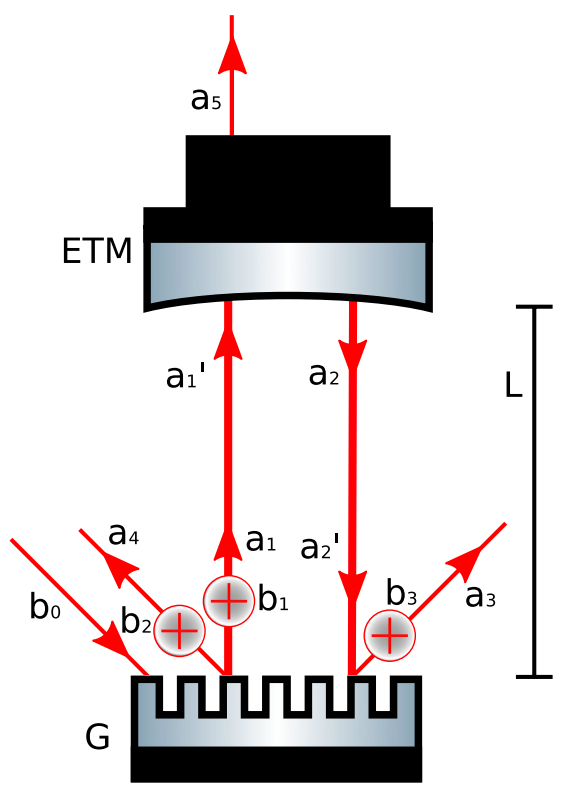

Figure 3. External input port $b_{0}$, internal input ports $b_{1}, b_{2}, b_{3}$ (marked by encircled addition symbols) and transfer ports $a_{q}$ in a grating cavity.

First order diffraction $(\mu=1)$ occurs between $a_{2}^{\prime}$ and $a_{4}$, so the sideband amplitude obtained is

$$
\mathrm{i} a_{2}^{\prime} \frac{\pi \Delta x}{d} G_{1}
$$

\subsection{Coupling relations of an oscillating grating as a Fabry-Perot cavity input optic}

To determine the transfer function for the generated noise sidebands to the output ports $a_{3}, a_{4}$ and $a_{5}$, and the input carrier field to ports $b_{1}, b_{2}$ and $b_{3}$ (in figure 3 ), it is necessary to know the port to port coupling relations for our three-port-coupled diffractive cavity of interest. Hence the coupling relations for the end-mirror ETM with amplitude transmission coefficient $\tau$ and amplitude reflection coefficient $\rho$ must be known,

$$
\left(\begin{array}{l}
a_{2} \\
a_{5}
\end{array}\right)=\left[\begin{array}{cc}
\rho_{2} & \mathrm{i} \tau_{2} \\
\mathrm{i} \tau_{2} & \rho_{2}
\end{array}\right]\left(\begin{array}{c}
a_{1}^{\prime} \\
0
\end{array}\right)
$$


and for the space of length $L$ between the grating and mirror,

$$
\left(\begin{array}{l}
a_{1}^{\prime} \\
a_{2}^{\prime}
\end{array}\right)=\left[\begin{array}{cc}
\mathrm{e}^{-\mathrm{i} k L} & 0 \\
0 & \mathrm{e}^{-\mathrm{i} k L}
\end{array}\right]\left(\begin{array}{l}
a_{1} \\
a_{2}
\end{array}\right),
$$

where $k=\omega / c$, with $\omega$ the angular frequency and $c$ the speed of light. Hence it is possible to write the local coupling relations for the diffractive cavity shown in figure 3 including the internal inputs discussed in section 3.2 at ports $b_{1}, b_{2}$ and $b_{3}$ :

$$
\begin{aligned}
& a_{1}=b_{0} G_{1}+a_{2}^{\prime} \rho_{0}+b_{1} \\
& a_{1}^{\prime}=a_{1} \mathrm{e}^{-\mathrm{i} k L} \\
& a_{2}=a_{1}^{\prime} \rho_{2} \\
& a_{2}^{\prime}=a_{2} \mathrm{e}^{-\mathrm{i} k L} \\
& a_{3}=b_{0} G_{0}+a_{2}^{\prime} G_{1}+b_{3} \\
& a_{4}=b_{0} G_{2}+a_{2}^{\prime} G_{1}+b_{2} \\
& a_{5}=a_{1}^{\prime} \mathrm{i} \tau_{2} .
\end{aligned}
$$

For the purposes of simplification, we define the resonance term of the cavity:

$$
D(\omega)=\frac{1}{1-\rho_{2} \rho_{0} \mathrm{e}^{-2 \mathrm{i} k L}} .
$$

By performing some substitution we obtain the port-to-port relations only in terms of the input ports $b$ and the fixed cavity parameters:

$$
\begin{gathered}
a_{1}=\left(b_{0} G_{1}+b_{1}\right) D(\omega) \\
a_{1}^{\prime}=\left(b_{0} G_{1}+b_{1}\right) D(\omega) \mathrm{e}^{-\mathrm{i} k L} \\
a_{2}=\left(b_{0} G_{1}+b_{1}\right) D(\omega) \rho_{2} \mathrm{e}^{-\mathrm{i} k L} \\
a_{2}^{\prime}=\left(b_{0} G_{1}+b_{1}\right) D(\omega) \rho_{2} \mathrm{e}^{-2 \mathrm{i} k L} \\
a_{3}=b_{0} G_{0}+\left(b_{0} G_{1}+b_{1}\right) D(\omega) \rho_{2} \mathrm{e}^{-2 \mathrm{i} k L} G_{1}+b_{3} \\
a_{4}=b_{0} G_{2}+\left(b_{0} G_{1}+b_{1}\right) D(\omega) \rho_{2} \mathrm{e}^{-2 \mathrm{i} k L} G_{1}+b_{2} \\
a_{5}=\left(b_{0} G_{1}+b_{1}\right) D(\omega) \mathrm{e}^{-\mathrm{i} k L} \mathrm{i} \tau_{2} .
\end{gathered}
$$

\subsection{Carrier field solution}

From the carrier amplitude present at $a_{2}^{\prime}$ we can determine the sideband amplitudes at $b_{1}, b_{2}$ and $b_{3}$. To obtain the carrier amplitude at $a_{2}^{\prime}$ it is necessary to solve equation (16) with an example input field at port $b_{0}$ of arbitrary angular frequency and amplitude $p_{0} \mathrm{e}^{-\mathrm{i} \omega_{\mathrm{c}} t}$ and no field at the internal input ports $b_{1}, b_{2}, b_{3}$, hence obtaining

$$
a_{2}^{\prime}=p_{0} G_{1} D\left(\omega_{\mathrm{c}}\right) \rho_{2} \mathrm{e}^{-2 \mathrm{i} k_{\mathrm{c}} L},
$$

where $a_{2}^{\prime}$ is the complex field amplitude of the carrier field. Since this solution is specific to the carrier, we distinguish it by using the subscript $\mathrm{c}$, and perform some simplification by introducing $B_{\mathrm{c}}=D\left(\omega_{\mathrm{c}}\right) \rho_{2} \mathrm{e}^{-2 \mathrm{i} k_{\mathrm{c}} L}$ :

$$
a_{2}^{\prime}=p_{0} G_{1} B_{\mathrm{c}}
$$

\subsection{Input sideband fields}

There is now sufficient information to determine the sideband field amplitudes $b_{1}, b_{2}$ and $b_{3}$. These are the linear sums of the sidebands created by the oscillating grating with the impinging fields in $b_{0}$ and $a_{2}^{\prime}$. Using equations (8)-(11) and (21) we can now write

$$
\begin{gathered}
b_{1}=\mathrm{i} p_{0} G_{1} \frac{\pi \Delta x}{d}, \\
b_{2}=\mathrm{i} p_{0} G_{2} \frac{2 \pi \Delta x}{d}+\mathrm{i} p_{0} G_{1}^{2} B_{\mathrm{c}} \frac{\pi \Delta x}{d}, \\
b_{3}=-\mathrm{i} p_{0} G_{1}^{2} B_{\mathrm{c}} \frac{\pi \Delta x}{d},
\end{gathered}
$$

where an input field of amplitude $p_{0}$ at port $b_{0}$ has been used.

\subsection{Sideband fields at outputs}

To determine the sideband field amplitude at the outputs $a_{3}$, $a_{4}$ and $a_{5}$ we consider the total field at the outputs (given by equations (17)-(19)). Setting the external input at port $b_{0}$ to zero leaves the internal inputs which are expressions of the sidebands. Equations (22)-(24) for the internal inputs are substituted into these output field equations, allowing them to be compared in terms of the fixed cavity properties and $p_{0}$. The sideband fields have different frequencies $\left(\omega_{\mathrm{u}}=\omega_{\mathrm{c}}+\omega_{m}\right.$ for the upper sideband, $\omega_{1}=\omega_{\mathrm{c}}-\omega_{m}$ for the lower sideband) and hence we address them separately, upper sideband first:

$$
\begin{gathered}
a_{3}=\mathrm{i} p_{0} G_{1}^{2} \frac{\pi \Delta x}{d}\left(B_{\mathrm{u}}-B_{\mathrm{c}}\right), \\
a_{4}=\mathrm{i} p_{0} G_{1}^{2} \frac{\pi \Delta x}{d}\left(B_{\mathrm{u}}+B_{\mathrm{c}}\right)+\mathrm{i} p_{0} G_{2} \frac{2 \pi \Delta x}{d}, \\
a_{5}=-p_{0} G_{1} \frac{\pi \Delta x}{d} \frac{B_{\mathrm{u}}}{\rho_{2} \mathrm{e}^{-\mathrm{i} k_{\mathrm{u}} L}} \tau_{2} .
\end{gathered}
$$

$a_{3}, a_{4}$ and $a_{5}$ are complex amplitudes associated with the upper sideband at each output, whilst $p_{0}$ is the complex amplitude for the carrier field input in the port $b_{0} . \omega_{\mathrm{u}}$ is the absolute frequency of the upper sideband and $B_{\mathrm{u}}=D\left(\omega_{\mathrm{u}}\right) \rho_{2} \mathrm{e}^{-\mathrm{i} k_{\mathrm{u}} L}$, analogous to our previously defined $B_{\mathrm{c}}$. The calculation can be repeated to show that the lower sideband has the same magnitude as the upper at all output ports. We have thus computed the optical signal in all output ports generated by lateral motion of the grating.

\section{Signal coupling in a grating cavity}

In this section we consider the interaction between the cavity and a potential gravitational wave propagating perpendicular to the cavity axis. The gravitational wave imposes phase modulation sidebands onto the light field inside the cavity.

The modulation index of the gravitational wave can be expressed in terms of an equivalent displacement of the mirror $\Delta z$ resulting in modulation index $m=4 \pi \Delta z / \lambda$. The effect of this displacement appears at cavity internal input $b_{1}$ in figure 3 with no input in ports $b_{2}$ and $b_{3}$. The port-to-port coupling relations given in section 3.3, and specifically equations (17)(19) for the output ports, can be used since the cavity coupling 
relations are unaffected by the case chosen (displaced grating or incident gravitational wave).

The carrier field amplitude $a_{2}^{\prime}$ can still be used from equation (21) as the carrier field is by definition unaffected when changing from the displaced grating to the incident gravitational wave case. Hence from the modulation index and the carrier field present, again using the example input field of amplitude $p_{0}$, we obtain the internal input amplitude:

$$
b_{1}=\mathrm{i} p_{0} G_{1} B_{\mathrm{c}} \frac{2 \pi \Delta z}{\lambda} \text {. }
$$

Therefore the output amplitudes at the upper sideband frequency are

$$
\begin{gathered}
a_{3}=\mathrm{i} p_{0} G_{1}^{2} \frac{2 \pi \Delta z}{\lambda} B_{\mathrm{c}} B_{\mathrm{u}}, \\
a_{4}=\mathrm{i} p_{0} G_{1}^{2} \frac{2 \pi \Delta z}{\lambda} B_{\mathrm{c}} B_{\mathrm{u}}, \\
a_{5}=-p_{0} G_{1} \frac{2 \pi \Delta z}{\lambda} \frac{B_{\mathrm{c}}}{\rho_{2} \mathrm{e}^{-\mathrm{i} k_{\mathrm{c}} L}} \frac{B_{\mathrm{u}}}{\rho_{2} \mathrm{e}^{-\mathrm{i} k_{\mathrm{u}} L}} \tau_{2} .
\end{gathered}
$$

We have thus computed the optical signal in all output ports due to a potential gravitational wave signal.

\section{Ratio of signal to noise at the outputs of a grating cavity}

The ratio of gravitational wave signal to lateral grating displacement noise (the signal to noise ratio) will be used as a figure of merit to evaluate the interferometric length sensing performance of the different diffractive cavity output ports. In order to derive the SNR the absolute field amplitude in the gravitational wave case will be divided by the absolute field amplitude in the grating lateral displacement case for each output $^{4}$. Using equation (25)-(27) and (29)-(31) we obtain the following signal to noise ratios at the output port of the equivalent subscript:

$$
\begin{gathered}
\mathrm{SNR}_{3}=\Lambda_{\mathrm{cav}} \frac{B_{\mathrm{u}}}{B_{\mathrm{u}}-B_{\mathrm{c}}}, \\
\mathrm{SNR}_{4}=\Lambda_{\mathrm{cav}} \frac{B_{\mathrm{u}}}{B_{\mathrm{u}}+B_{\mathrm{c}}+2 G_{2} / G_{1}^{2}}, \\
\mathrm{SNR}_{5}=\Lambda_{\mathrm{cav}} / \rho_{2} \mathrm{e}^{-\mathrm{i} k_{\mathrm{c}} L},
\end{gathered}
$$

with

$$
\Lambda_{\mathrm{cav}}=\frac{2 d \Delta z B_{\mathrm{c}}}{\lambda \Delta x} .
$$

These equations reveal the differences between the ratio of gravitational wave signal to lateral grating displacement noise for the three cavity output ports. This SNR is frequency independent for the case of the output port in transmission of the cavity end-mirror $\left(a_{5}\right)$, while the SNR at the other two output ports contains the modulation frequency dependent term $B_{\mathrm{u}}$.

4 Note that for a standard cavity with equivalent finesse, the SNR will be infinity as in principle two-mirror cavity input mirrors are insensitive to lateral displacement. In reality however in any suspension system there is always a coupling from lateral excitation to longitudinal displacement.
It was found that the SNR for the two output ports $a_{3}$ and $a_{4}$ includes the SNR from inside the cavity $\Lambda_{\text {cav }}$, multiplied by a fraction containing the different resonance factors for the carrier light field and the sidebands. Apart from the small $G_{2} / G_{1}^{2}$ term, the only difference between equations (32) and (33) is whether the resonance terms of the carrier and sideband in the denominator are added or subtracted. The important feature of these equations is that $B_{\mathrm{u}} \approx B_{\mathrm{c}}$ for small modulation frequencies (i.e. modulation frequencies within the cavity bandwidth). This brings the denominator in equation (32) close to zero, thus strongly increasing the SNR at port $a_{3}$, i.e. resulting in a partial cancellation of the phase noise introduced from lateral grating displacement.

\subsection{Numerical result}

In this section we present a quantitative analysis for one example configuration. We choose a cavity length of $L=$ $3 \mathrm{~km}, \rho_{2}=\sqrt{0.99995}$ (50 ppm power transmittance) and $\rho_{0}=\sqrt{0.95}$. Demanding that the $\mu=1$ diffraction order propagates normally to the grating for the input beam imposes the grating design requirement $d \leqslant 2 \lambda$ [13]. The grating phase relations further impose the minimum possible value of $\eta_{2} \geqslant 0.0127$. From [8] for minimum $\eta_{2}$ we find that $G_{2}=-0.0127$. $G_{1}$ is fixed by the required cavity input parameters. Together $G_{1}$ and $G_{2}$ set the value of the last term in the denominator in equation (33) and it is small compared to the other terms of the denominator. The cavity is set to resonance for the carrier light, imposing $\mathrm{e}^{-2 \mathrm{i} k_{\mathrm{c}} L}=1$, and the modulation frequency $\omega_{m}$ of the lateral grating displacement is chosen to be $10 \mathrm{~Hz}$.

Our interest focuses on the relative magnitude of the frequency sidebands. As in [6] we set $\Delta z / \Delta x=1$ in order to compare the potential gravitational wave signal to the effect of grating displacement. Since we are dealing with complex field amplitudes for this analysis we take the absolute value of the ratios ${ }^{5}$ given in equations (32)-(34), yielding the following result:

$$
\begin{gathered}
\left|\mathrm{SNR}_{3}\right|=3181 \\
\left|\mathrm{SNR}_{4}\right|=79 \\
\left|\mathrm{SNR}_{5}\right|=158 .
\end{gathered}
$$

In all output ports the SNR is found to be greater than one, as the cavity suppresses the displaced grating phase noise sidebands with a factor of 158 as seen in the transmitted output port; however, a factor of 20 improvement in the SNR can be obtained through the cancellation of input and output grating displacement sidebands in the forward-reflected $\left(a_{3}\right)$ port compared to the transmitted $\left(a_{5}\right)$ port.

For proposed interferometric gravitational wave detector layouts the end mirror will be highly reflective ( $\rho_{2}$ will be close to one) and therefore the complex field amplitudes for the potential gravitational wave signal (equations (29)-(31)) will obey the relations $a_{3} \gg a_{5}, a_{4} \gg a_{5}$. There will be

5 The ratio contains a real part as well as an imaginary part. We assumed that the readout quadrature of each of the three output ports can be chosen individually by adding a proper local oscillator, i.e. performing a homodyne measurement. 


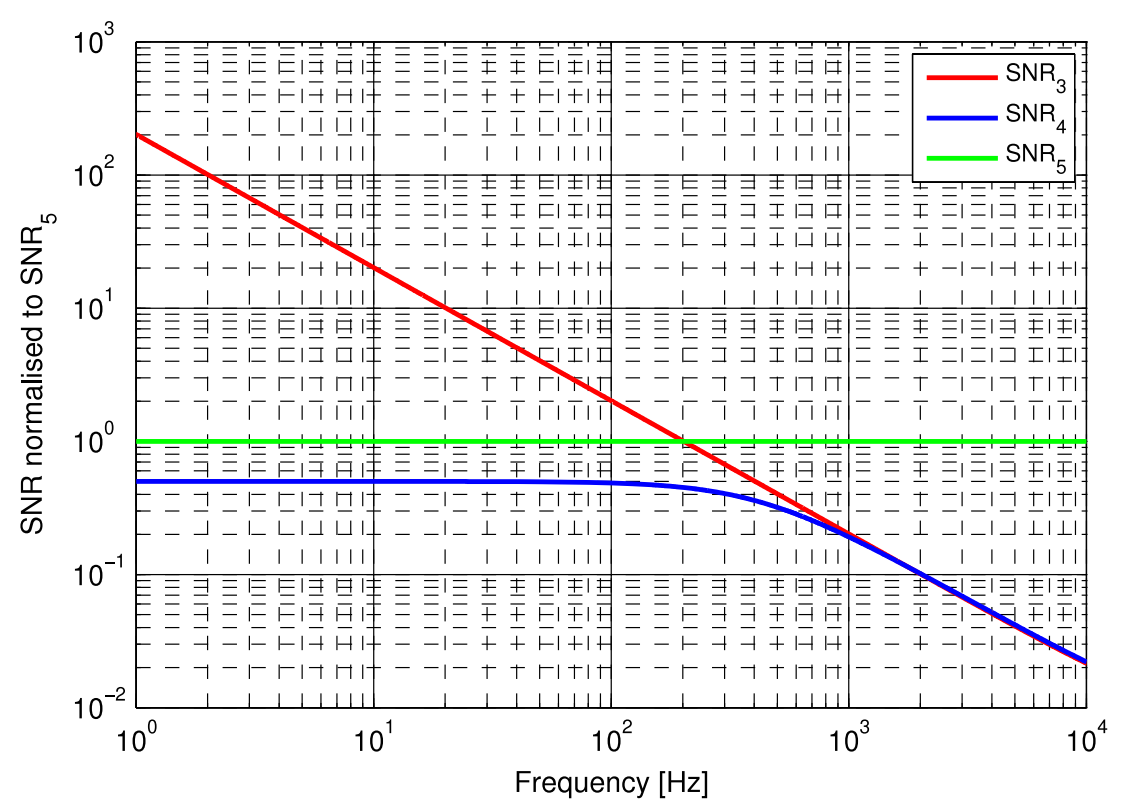

Figure 4. Signal to noise ratio of gravitational wave equivalent mirror displacement and lateral grating displacement at the three different output ports of a grating gravity. All traces are normalized to $\mathrm{SNR}_{5}$.

approximately half the signal in the forward-reflected port with good SNR, half will be in the back-reflected port with poor SNR and very little will be in the transmitted port.

In a next step, instead of a single frequency of interest, we consider modulation frequencies covering the full detection band of gravitational wave detectors. As $\mathrm{SNR}_{3}$ and $\mathrm{SNR}_{4}$ are frequency dependent, we normalize them to $\mathrm{SNR}_{5}$ and plot them over frequency to obtain figure 4.

At low modulation frequencies, where the sidebands are to a good approximation resonant in the cavity, imposing $B_{\mathrm{u}} \approx B_{\mathrm{c}}$ the forward-reflected port $\left(a_{3}\right)$ has good cancellation between the input and output sidebands generated by the grating lateral displacement. This results in high SNR compared to the transmitted port $\left(a_{5}\right)$. In the back-reflected port $\left(a_{4}\right)$ summation occurs instead of cancellation and hence the SNR is lower than in the transmitted port. As the modulation frequency diverges from the cavity resonance, the sidebands do not resonate in both the gravitational wave and grating lateral displacement case as $B_{\mathrm{u}}$ trends to zero. Thus the sideband contribution generated when the carrier field exits the cavity, the $B_{\mathrm{c}}$ terms in the denominator of equations (32) and (33), dominates, causing the SNR of the reflected ports to converge below the level of the transmitted port.

\subsection{Suspension requirements}

To ease comparison of the results derived above we determine the potential suspension requirements for a grating cavity used as an arm cavity within the planned Advanced Virgo detector. Using the current Advanced Virgo design sensitivity [14] we plot in figure 5 the corresponding tolerable lateral grating motion for each of the three potential readout ports of the grating arm cavity.

We have found that we can relax by a factor of 20 at $10 \mathrm{~Hz}$ (figure 5) the suspension requirement for the unsuppressed transmitted $\left(a_{5}\right)$ port by utilizing the $a_{3}$ (forwardreflected) port for the signal readout. This suppression depends on cancellation between noise sidebands which we have calculated for the case of the lateral suspension of the grating. It is likely that this suppression will also apply to the input pointing (as misaligned input pointing also changes the alignment of the field circulating in the cavity [15]) and hence the alignment stability requirement for the injection optics. However, sidebands due to end-mirror angular misalignment are not suppressed.

Freise et al [6] found that without suppression of the grating lateral displacement, using a three-port-coupled grating cavity rather than a standard two-mirror cavity, in the Advanced Virgo case, requires improving the end-mirror angular alignment suspension requirement by five orders of magnitude. The equivalent improvement required for the injection optics alignment stability and grating lateral suspension can be relaxed by a factor of 20 by choosing the forward-reflected port for the signal readout, at least in the lower frequency band.

Above $200 \mathrm{~Hz}$ this geometrical effect no longer suppresses coupling of lateral grating displacement in the forwardreflected port. In this case it is preferred to use the transmitted port for readout; however, the fixed SNR set by the cavity properties is not beaten, and the system is no longer entirely reflective.

The encouraging results presented above increase the application prospects of grating coupled Fabry-Perot cavities in large-scale gravitational wave detectors, but the problem raised by Freise et al of end-mirror angular alignment remains.

\section{Summary and outlook}

Diffractive optics are an important avenue of research because they allow all-reflective interferometer configurations which 


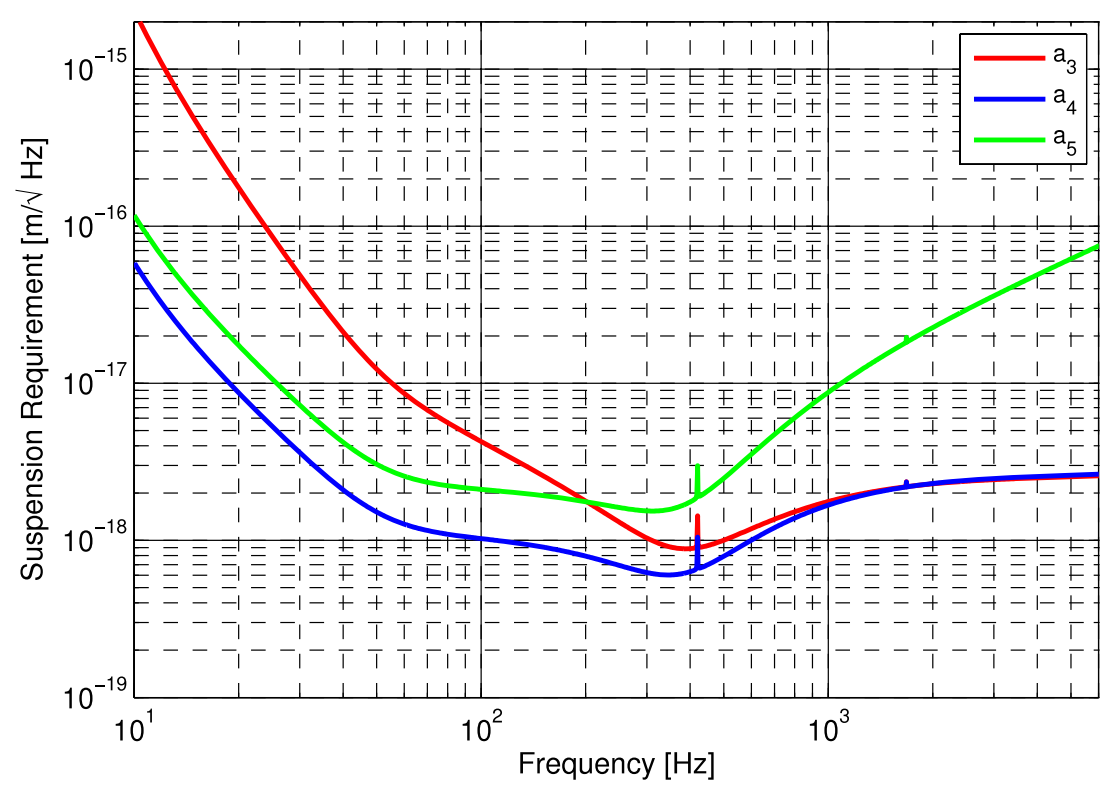

Figure 5. Suspension requirement for the maximum tolerable lateral grating displacement that is necessary to achieve the Advanced Virgo design sensitivity. The suspension required strongly depends on the actual readout port as well as the frequency of interest. At low frequencies the phase noise suppression in port $a_{3}$ allows significant relaxation of the required suspension isolation. The narrow peak around $410 \mathrm{~Hz}$ is due to a resonance in the Advanced Virgo design, not a grating effect.

offer thermal noise improvements and may be especially relevant in mitigating effects made important when high power is used to reduce shot-noise contribution. An additional noise source is lateral grating displacement relative to the beam causing path-length differences that couple phase noise into the gravitational wave detection channel. From [6] the gratingcavity end-mirror angular alignment suspension requirement (five orders of magnitude higher than that required by a twomirror cavity), and associated likely increases in injection optic pointing and lateral grating stability, were a significant impetus against using grating cavities.

The diffraction order $(\mu)$ dependence of the frequency sideband amplitude and the symmetry inherent in the threeport-coupled grating cavity suggested that the phase noise generated on input to the cavity might cancel with the phase noise generated at output from the cavity.

To determine the effect we carried out a frequency domain analysis to calculate the coupling of lateral grating displacement to the different output ports of a three-portcoupled grating cavity. For the output port in forward reflection of the grating we found a suppression of phase noise originating from lateral grating displacement over the transmitted port, resulting in a factor of 20 relaxation in the lateral displacement suspension requirement at $10 \mathrm{~Hz}$. This will likely also apply to the injection optics pointing stability. This factor will increase with a wide cavity bandwidth and hence the noise suppression will be greater in cavities of lower finesse.

\section{Acknowledgments}

This work was supported by the Science and Technology Facilities Council (STFC), the European Gravitational Observa- tory (EGO) and the Deutsche Forschungsgemeinschaft (DFG) within the Sonderforschungsbereich (SFB) TR7.

\section{References}

[1] Sun K X and Byer R L 1998 All-reflective Michelson, Sagnac, and Fabry-Perot interferometers based on grating beam splitters Opt. Lett. 23 567-9

[2] Drever R W P 1996 Concepts for extending the ultimate sensitivity of interferometric gravitational wave detectors using non-transmissive optics with diffractive or holographic coupling Proc. 7th Marcel Grossman Mtg on Recent Developments in Theoretical and Experimental General Relativity, Gravitation, and Relativistic Field Theories p 1401

[3] Bunkowski A, Burmeister O, Beyersdorf P, Danzmann K, Schnabel R, Clausnitzer T, Kley E-B and Tünnermann A 2004 Low-loss grating for coupling to a high-finesse cavity Opt. Lett. 29 2342-4

[4] Bunkowski A, Burmeister O, Clausnitzer T, Kley E-B, Tünnermann A, Danzmann K and Schnabel R 2006 Optical characterization of ultrahigh diffraction efficiency gratings Appl. Opt. 45 5795-9

[5] Perry M D, Boyd R D, Britten J A, Decker D, Shore B W, Shannon C and Shults E 1995 High-efficiency multilayer dielectric diffraction gratings Opt. Lett. 20 940-2

[6] Freise A, Bunkowski A and Schnabel R 2007 Phase and alignment noise in grating interferometers New J. Phys. 9433

[7] Acernese F et al 2008 Virgo status Class. Quantum Grav. 25184001

[8] Bunkowski A, Burmeister O, Danzmann K and Schnabel R 2005 Input-output relations for a three-port grating coupled Fabry-Perot cavity Opt. Lett. $301183-5$

[9] Freise A 2008 FINESSE 0.99.8 User Manual University of Birmingham http://www.gwoptics.org

[10] Freise A 2003 The next generation of interferometry: multi-frequency optical modelling, control concepts and implementation PhD Thesis Universität Hannover http:// www.sr.bham.ac.uk/ adf/space/publications.php 
[11] Heinzel G 1999 Advanced optical techniques for laser-interferometric gravitational-wave detectors PhD Thesis MPI fuer Quantenoptik http://en. scientificcommons.org/34745751

[12] Mizuno J and Yamaguchi I 1999 Method for analyzing multiple-mirror coupled optical systems J. Opt. Soc. Am. A 16 1730-9

[13] Clausnitzer T, Kley E-B, Tünnermann A, Bunkowski A, Burmeister O, Danzmann K, Schnabel R, Gliech S and
Duparré A 2005 Ultra low-loss low-efficiency diffraction gratings Opt. Express 134370

[14] Hild S, Losurdo G and Freise A 2009 Sensitivity curves for the Advanced Virgo preliminary design Technical Report VIR-101A-08 (Virgo) https://pub3.ego-gw.it/codifier/ [15] Morrison E, Robertson D I, Ward H and Meers B J 1994 Experimental demonstration of an automatic alignment system for optical interferometers Appl. Opt. 33 5037-40 